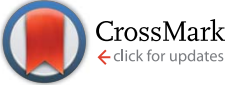

Cite this: RSC Adv., 2017, 7, 12753
Received 8th September 2016 Accepted 21st January 2017

DOI: $10.1039 / \mathrm{c} 6 \mathrm{ra22521g}$

rsc.li/rsc-advances

\section{A partial proteome reference map of Tetragenococcus halophilus and comparative proteomic and physiological analysis under salt stress $\uparrow$}

\begin{abstract}
Guiqiang He, ${ }^{a b}$ Jingcheng Deng, ${ }^{\text {ab }}$ Chongde $W^{* a b}$ and Jun Huang ${ }^{a b}$
Tetragenococcus halophilus, a moderately halophilic Gram-positive lactic acid bacteria, was widely existed in many food fermentation systems, where salt stress is an environmental condition commonly encountered. In this study, a partial proteome reference map of T. halophilus was established by using the proteomics approach. A total of 120 proteins were identified and the cluster of orthologous groups (COG), codon adaptation index (CAI), and GRAVY value analyses of the identified proteins were performed. To better understand the salt tolerance mechanisms and stress responses, we carried out a comparative proteomic and physiological study of $T$. halophilus during salt stress. Comparative proteomic analysis revealed 26 differentially expressed proteins that are mainly involved in cellular metabolism, energy production, stress response, and others. Analysis of the cytoplasmic membrane fatty acids showed that higher proportions of unsaturated fatty acid and higher mean chain length were observed in the cells challenged by salt stress. In addition, the salt shocked cells exhibited higher amounts of intracellular proline, glycine betaine, and trehalose compared with those without salt stress. Results presented in this study may further elucidate the mechanisms of $T$. halophilus underlying salt stress.
\end{abstract}

\section{Introduction}

Tetragenococcus halophilus is a moderately halophilic Grampositive lactic acid bacterium found in many fermented foods, mostly highly salted fermented products, such as soy sauce mash $^{\mathbf{1 , 2}}$ and fish sauce. ${ }^{3,4}$ It plays important roles in fermentation processes during the production of pickled products. For example, previous research studies demonstrated that addition of $T$. halophilus as a starter culture for soy sauce moromi or fish sauce fermentation could promote the formation of volatile compounds and improve the flavor characteristics. ${ }^{3,5}$ However, T. halophilus was inevitably subjected to salt stress during the manufacture of these salted foods. Generally, exposure of cells to high salinity environments may lead to structural and physiological injury of cells and a decrease in metabolic activities and production efficiency.

Therefore, developing adaptive strategies to cope with salt stress is essential for cells to exert their functional characteristics in food fermentation. ${ }^{6}$ In response to salt stress, like other

${ }^{a}$ College of Light Industry, Textile \& Food Engineering, Sichuan University, Chengdu 610065, China. E-mail: cdwu@scu.edu.cn; Fax: +86-28-85405237; Tel: +86-2885406149

${ }^{b}$ Key Laboratory of Leather Chemistry and Engineering, Ministry of Education, Sichuan University, Chengdu 610065, China

$\dagger$ Electronic supplementary information (ESI) available. See DOI: 10.1039/c6ra22521g bacteria, lactic acid bacteria (LAB) have acquired an amazing variety of adaptation and defense mechanisms to cope with harmful effects and to survive in hostile environments. Intracellular accumulating of osmoprotectants is one of most effective strategies of $\mathrm{LAB}$ responding to salt stress, which can equilibrate cellular osmotic pressure. ${ }^{7}$ Modification of the cytoplasmic membrane fatty acid compositions to withdraw the injury from osmotic stress is another powerful strategy. ${ }^{\mathbf{8}}$

In recent years, omics technologies have paved the way for the studies of adaptive response of bacterium to salt stress., ${ }^{\mathbf{9} 10}$ Zhang et al. ${ }^{11}$ established a reference proteome map of intracellular proteins of Lactococcus lactis SK11 using a proteomics approach, and a comparative proteomic analysis was carried out to identify and characterize proteins expressed in the cells containing or not containing glutathione under salt stress. Moreover, omics technologies for the manipulation of gene expression levels are available, allowing complementary approaches to alter expression levels of genes encoding stress response proteins. For example, the heterologous expression of RecO protein in Lactococcus lactis, resulted in a significant higher tolerance to multiple-stress and enhanced lactic acid production during salt stress. ${ }^{12}$ Yet another example revealed that expression of PprI from Deinococcus radiodurans improved oxidative stress and osmotic stress tolerance in L. lactis. ${ }^{13}$

Recently, Liu et al. ${ }^{\mathbf{1 4}}$ investigated salt tolerance mechanisms of T. halophilus by transcriptomic analysis, and results showed 
that genes involved in transcription, translation, membrane system, and division were highly up-regulated under salt stress. Acid tolerance response of T. halophilus was also investigated based on physiological and proteomic analysis, and proteomic analysis demonstrated that eight proteins involved in carbohydrate metabolism and three classic stress response proteins were up-regulated after acid adaptation. ${ }^{15}$ Despite the importance roles of $T$. halophilus, little is known about its protein expression in response to salt stress. Thus, the aim of this study was to investigate the salt stress response of T. halophilus via the physiological analysis. Results presented in this study may contribute to understand the salt tolerance mechanisms and lay the foundation for improving salinity resistance of $T$. halophilus.

\section{Materials and methods}

\subsection{Strains and salt stress experiment}

Tetragenococcus halophilus CGMCC 3792, which was isolated from soy sauce moromi and identified by $16 \mathrm{~S}$ rDNA sequence, ${ }^{1}$ was used in this study. The cells were cultivated statically in GM17 (M17 broth (OXOID, UK) supplemented with 0.5\% (w/v) glucose) at $30{ }^{\circ} \mathrm{C}$ for $20 \mathrm{~h}$ to obtain a pre-culture. To investigate the salt tolerance of $T$. halophilus, the pre-culture was inoculated $(5 \%, v / v)$ into a fresh GM17. Cells grown to midexponential growth phase were collected, centrifugated $(10000 \times g, 5 \mathrm{~min})$, washed, and resuspended with modified GM17 containing different concentrations of $\mathrm{NaCl}$ for $2 \mathrm{~h}$ at $30{ }^{\circ} \mathrm{C}$. After salt stress, cells were serially diluted, plated onto GM17 agar plates, and incubated at $30^{\circ} \mathrm{C}$ for $48 \mathrm{~h}$. Cell survival rate was expressed as the percentage of viable cells (CFU per milliliter).

\subsection{Organic acid analysis}

Cells cultivated in GM17 supplemented with different concentrations of $\mathrm{NaCl}$ were harvested, centrifugated $(10000 \times g, 5$ $\mathrm{min}$ ), and the supernatants were acidified by addition of $5 \mathrm{mM}$ $\mathrm{H}_{2} \mathrm{SO}_{4}$. Supernatants were then filtered with a $0.22 \mu \mathrm{m}$ membrane filter, the filtrate was analyzed by high-pressure liquid chromatography (HPLC, Agilent 1200, which was fitted with an organic acid column $(300 \mathrm{~mm} \times 4.6 \mathrm{~mm}$, OA-1000, Alltech, USA), USA) according to the method of Tiina Michelson et al. ${ }^{16}$

\subsection{Intracellular amino acid analysis}

For the extraction of intracellular amino acids, $10 \mathrm{~mL}$ of cultures at an $\mathrm{OD}_{600}$ of 2.5 was harvested, centrifugated (10 000 $\times g$, $5 \mathrm{~min}$ ), washed, resuspended in $1 \mathrm{~mL}$ of $200 \mathrm{mM}$ phosphate buffer saline (PBS, pH 7.0), and boiled for $15 \mathrm{~min}$. Cell debris was discarded by centrifugation $\left(12000 \times g, 10 \mathrm{~min}, 4^{\circ} \mathrm{C}\right)$. The supernatants were treated at ambient temperature by addition of $100 \mu \mathrm{L} 10 \%(\mathrm{w} / \mathrm{v})$ sulphosalicylic acid for $10 \mathrm{~min}$. The mixture was then centrifugated as noted again and the supernatants were filtered with a $0.22 \mu \mathrm{m}$ filter. Sample solutions with $20 \mu \mathrm{L}$ were detected for amino acid composition using an amino acid analyzer (A300, membraPure $\mathrm{GmbH}$, Germany) according to the method of Cui et al. ${ }^{5}$

\subsection{Intracellular osmoprotectants analysis}

Cells cultivated in GM17 with different $\mathrm{NaCl}$ concentrations were harvested, centrifugated $(10000 \times g, 5 \mathrm{~min})$, and washed with isotonic solution. For determination of glycine betaine, cells were resuspended in $1 \mathrm{~mL}$ of $50 \mathrm{mM}$ PBS (pH 7.0), and then disrupted ultrasonically in iced bath for 100 cycles of $5 \mathrm{~s}$ (JY92IIN sonicator, China). After sonication, the extraction solutions were immediately centrifugated $\left(10000 \times g, 5 \mathrm{~min}, 4{ }^{\circ} \mathrm{C}\right)$, and the supernatants were analyzed following the previous method. ${ }^{17}$ Trehalose was extracted and assayed according to the previous literature. ${ }^{18}$

\subsection{Membrane fatty acid analysis}

Extraction of membrane lipids and preparation of fatty acid methyl esters (FAMEs) were carried out according to the method of $\mathrm{Wu}$ et $a .^{19}$ Analysis of the samples was performed by gas chromatography-mass spectrometer (GC-MS, Trace GC Ultra-DSQ II, Thermo Electron Corporation, USA) according to the method described in the literature. ${ }^{20}$ The relative amount of FAMEs was calculated from peak areas. The degree of unsaturation (unsaturated fatty acids/saturated fatty acid, U/S ratio) and the mean chain length were assayed according to the method described previously. ${ }^{19}$ All experiments were carried out in triplicate.

\subsection{Protein preparation, $2 \mathrm{D}$ gel electrophoresis, and image analysis}

Cells collected at exponential phase $\left(20 \mathrm{~mL}, \mathrm{OD}_{600}=2.5\right)$ were centrifugated $(10000 \times g, 5 \mathrm{~min})$, washed, and then resuspended in GM17 supplemented with $12 \% \mathrm{NaCl}$. After shocked for $2 \mathrm{~h}$, the cells were washed to remove the residual medium. The whole cell extracts were prepared using the BioRad Kit. Briefly, the washed cells were lysed in lysis buffer ( $8 \mathrm{M}$ urea, $4 \%$ CHAPS, $30 \mathrm{mM}$ Tris $\mathrm{pH} 8.5$ ), and the solution was sonicated on ice for $20 \mathrm{~min}$ and followed by centrifugation at $16000 \times g$ for $30 \mathrm{~min}$. Proteins in the supernatant were treated with the Cleanup kit (BioRad) and dissolved in lysis buffer and treated by

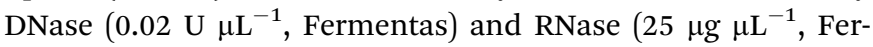
mentas). The protein concentration was determined by using the BioRad Protein Assay Kit with BSA as a standard. The sample was applied to immobilized pH gradient (IPG) strips (18 cm, pH 4-7, BioRad) with a final concentration of $100 \mu \mathrm{g}$ protein in $350 \mu \mathrm{L}$ rehydration buffer ( $8 \mathrm{M}$ urea, $2 \%(\mathrm{w} / \mathrm{v})$ CHAPS, $20 \mathrm{mM}$ DTT, $0.5 \%$ (v/v IPG buffer), $0.002 \%$ bromphenol blue). Isoelectric focusing (IEF) of the protein sample was performed according to the method described previously. ${ }^{21}$ After IEF, the IPG strips were equilibrated sequentially in a buffer (Tris-HCl contained $6 \mathrm{M}$ urea, 30\% glycerol, and 2\% SDS) that contained $1 \%$ DTT or $2.5 \%$ iodoacetamide for $15 \mathrm{~min}$ each. The equilibrated IPG strips were run on $12 \%$ SDS polyacrylamide gels. Electrophoresis was performed using an Ettan DALT-12 unit (GE Healthcare) according to the method described by $\mathrm{Wu}$ et $a .^{21}$ After SDS-PAGE, the 2D gels were stained with $0.1 \%$ coomassie blue R-250 (Sangon Biotech, China).

The stained gels were scanned using Imagescanner (GE Healthcare) at $300 \mathrm{dpi}$ resolution. The images were analyzed 
using Image Master 6.0 2D platinum software (GE Healthcare). The spot detection, gel matching, and spot quantification were performed by using the automatic mode as previously described, followed by manual checking. ${ }^{22}$ Only protein spots showing at least 2-fold change were considered for statistic analysis (Student's $t$ test). Significant differentially expressed protein spots $(p<0.05)$ were considered for further analysis.

\subsection{In-gel digestion, MS analysis, and protein identification}

Proteins spots were excised using gel plugs, transferred to Eppendorf tubes, then in-gel digestion by trypsin. ${ }^{22}$ Supernatants of $0.5 \mu \mathrm{L}$ were spotted directly onto the MALDI plate (containing 0.4 $\mu \mathrm{L} \mathrm{CHCA}$ ), and samples were analyzed on the Applied Biosystem 4700 Proteomics Analyzer MALDI-TOF/TOF (Applied Biosystmes, Framingham, MA) in positive ion reflector mode. MS and MS/MS data were analyzed and peak lists were generated using GPS Explorer v3.5 (Applied Biosystmes). MS peaks were selected between 700 and 4000 Da and filter was used with a signal-to-noise ratio greater than 20 . Database searches for MS or MS/MS spectra were performed by using the Mascot 2.0 to search against the T. halophilus protein sequence database, NCBInr, and Swissprot database. The searching parameters were set to use trypsin digestion with one missed cleavage, and use carbamidomethyl as a fixed modification of cysteine and oxidized methionine as a variable modification. The mass tolerance of precursor ion and fragment ion were set to $0.2 \mathrm{Da}$ for +1 charged ions. MS or MS/MS identifications were accepted when the protein score or total ion score were higher than 95.

\subsection{In silico analysis of the identified proteins}

The codon adaptation index (CAI) of all genes and grand average of hydropathy (GRAVY) of each protein of T. halophilus were generated by CodonW software as previously described. ${ }^{23}$ Experimentally identified proteins and all ORFs were grouped into cellular roles according to COGs as well (http:// www.ncbi.nlm,nih.gov/COG/). The theoretical 2-DE map of $T$. halophilus was constructed using the JVirGel 2.0 web service (http://www.jvirgel.de/). ${ }^{24}$

\subsection{Gene expression analysis by quantitative RT-PCR}

For the analysis of gene expression, $100 \mathrm{~mL}$ of mid-exponential growth phase cells was harvested, centrifugated $(10000 \times g$, 5 min, $4{ }^{\circ} \mathrm{C}$ ), washed, and stored at $-80{ }^{\circ} \mathrm{C}$ for further analysis. Extraction of total RNA, synthesis of first-strand cDNA, and qPCR experiments were performed according to the method of $\mathrm{Wu}$ et al. ${ }^{25}$ The expression levels of all the target genes were compared to the reference gene (16S rRNA). The $2^{-\Delta \Delta C_{t}}$ method was used to calculate the relative changes in gene expression level. ${ }^{26}$ The primers used for RT-PCR assay are listed in Table S1. $\dagger$

\subsection{Statistical analysis}

In this study, each analysis was carried out in triplicate. Analysis of variance (one-way ANOVA) with Duncan's test was performed to evaluate statistical differences. Differences between samples with $p$-values $p \leq 0.05(n=3)$ were considered to be statistically significant.

\section{Results}

\subsection{Effect of salt stress on the survival of $T$. halophilus}

The tolerance of $T$. halophilus subjected to different concentrations of $\mathrm{NaCl}(0,6,12,18,24,30$, and 36\%) was investigated, and the survival rates were determined (Fig. 1). As shown in Fig. 1, T. halophilus exhibited relatively broad ranges of $\mathrm{NaCl}$ tolerance and the survival rates decreased with the increase of $\mathrm{NaCl}$ concentration. When treated with $36 \% \mathrm{NaCl}$ for $2 \mathrm{~h}$, the survival rate decreased $83.3 \%$, compared with that without salt stress. Generally, T. halophilus has been frequently found in many high salt fermented foods, and this bacterium could grow in $18 \% \mathrm{NaCl}$ or greater. ${ }^{3,27,28}$

\subsection{2-DE proteome reference map of $T$. halophilus}

T. halophilus cells were subjected to 2-DE analysis to construct the proteome reference map. According to the genome annotation of T. halophilus, a total of 2587 predicted proteins were encoded. All of the predicted proteins were used to generate the theoretical 2-DE map by using the software JVirGel 2.0 (http:// www.jvirgel.de/), and about $70 \%$ of the proteins possess $\mathrm{pI}$ values between 4 and 7 (Fig. S1 $\dagger$ ). Therefore, IPG strip of $\mathrm{pH} 4-7$ was used for the first dimension separation of the cytoplasmic proteins. The representative 2-DE gel stained with coomassie blue was selected to serve as the reference map and was shown in Fig. 2. A total of 120 spots representing 116 proteins were successfully identified, and the numbers corresponding to the identified proteins were listed in Table $\mathbf{S} 2 . \dagger$

Based on the characterizations of identified proteins, the physico-chemical analysis was performed (Fig. 3). Experimentally identified proteins were grouped into cellular roles according to COGs (Clusters of Orthologous Groups) categories on the basis of biological or biochemical function, and

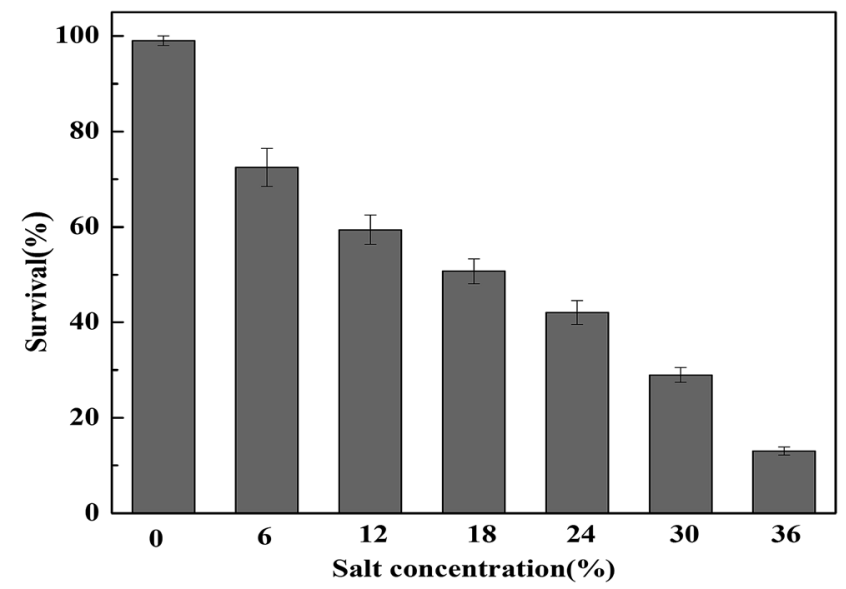

Fig. 1 Survival rates of $T$. halophilus under salt stress. Cultures were subjected to salt stress for $2 \mathrm{~h}$ and the survival rate was determined. Error bars indicate standard deviations $(n=3)$. 


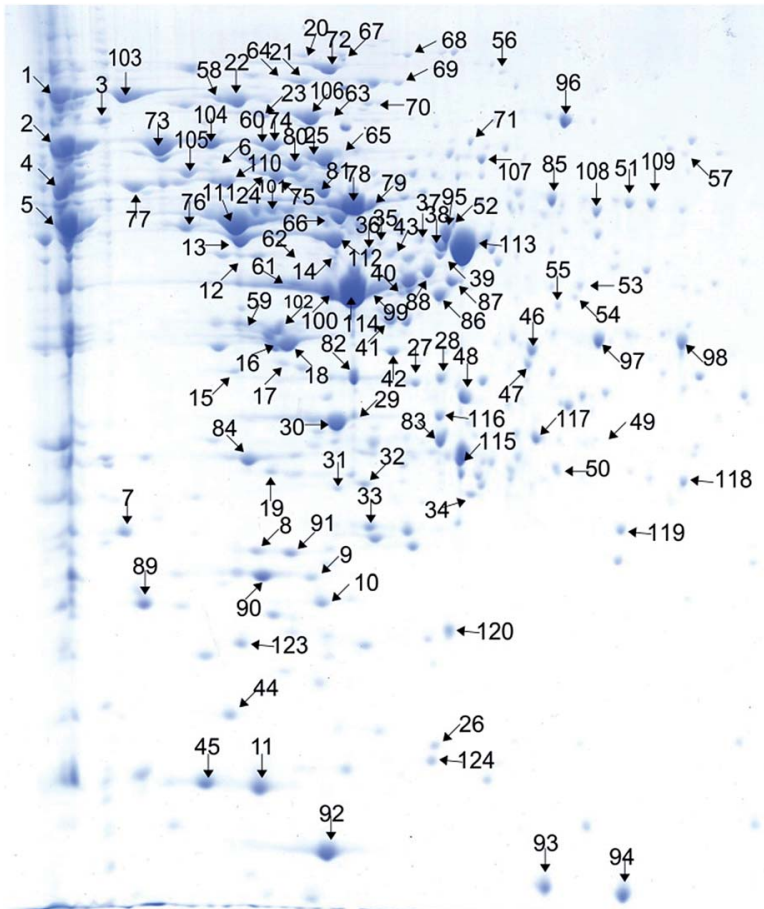

Fig. 2 Two-dimensional electrophoresis gels of the proteins of $T$. halophilus during salt stress. The identified proteins are marked by arrows and numbers and listed in Table S2.广

the distribution of COG category was presented (Fig. 3A). From Fig. 3A, the proteins involved in translation (J) and replication (L) comprised a great part of the identified proteins. Other abundant categories included proteins implicated in carbohydrate transport and metabolism (G) and transcription (K). The comparison of CAI (Codon Adaptation Index) distribution and GRAVY (grand average of hydrophobicity) index of the genes coding for the proteins identified on $\mathrm{pH} 4-7$ gels with those of the whole set of genes encoding proteins of $T$. halophilus were presented in Fig. 3B and C. The CAI value is used to determine the codon usage bias for a gene, and demonstrate the tendency of the corresponding protein synthesis. The proteins encoded by genes with a CAI value above 0.4 accounted for $94 \%$ of the total predicted proteins and $99 \%$ of the 120 identified proteins, respectively. This result suggests that proteins encoded by genes with a high CAI were easily identified. This observation is in agreement with previous results reported in Lactococcus lactis, ${ }^{23}$ Lactobacillus casei, ${ }^{29}$ and Bifidobacterium longum. ${ }^{30}$

The GRAVY index indicates the solubility of the proteins. By using the CodonW software, the GRAVY index was calculated for all of the theoretical and identified proteins. The comparison of GRAVY values revealed that all the identified proteins exhibited the GRAVY values lower than 0.2. The most hydrophobic protein identified in this work was the small heat shock protein $(-0.988)$. Only 3 identified proteins had a GRAVY index above zero, indicating that strongly hydrophobic proteins might be lost under the standard gel conditions. These results are similar to those demonstrated for other LAB. ${ }^{23,29,30}$
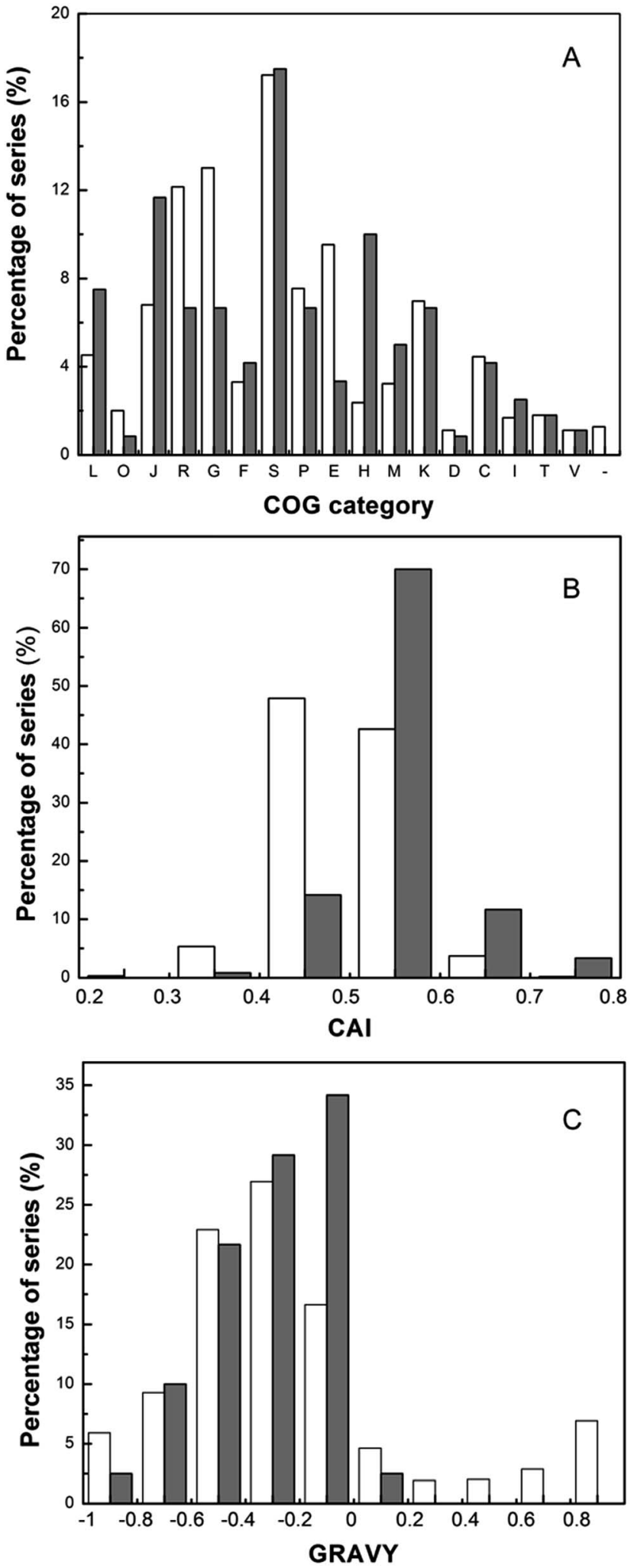

Fig. 3 Frequency distribution of the COG category, CAI, and GRAVY of the proteins. Based on the characterizations of identified proteins on a 2-DE gel in T. halophilus during salt stress, the physico-chemical ((A) COG category, (B) CAI, (C) GRAVY) analysis was performed. The open bars represent theoretical proteins, and the gray bars represent identified proteins. 


\subsection{Comparative proteomic analysis of $T$. halophilus during salt stress}

To investigate the salt stress response of $T$. halophilus, a comparative proteomic analysis was employed based on twodimensional gel electrophoresis followed by protein identification (Fig. S2†). Proteins that exhibited more than two-fold higher expression were taken into account for further analysis. The differentially expressed proteins were arranged by COG categories, and listed in Table 1. Analysis of the gels indicated that two proteins (TreB: spots 59 and GldA: 62) were produced exclusively during salt stress. Moreover, further analysis of the differentially expressed proteins showed that the majority of the proteins belonged to carbohydrate transport and metabolism (6 proteins), energy production and conversion (5), translation, ribosomal structure, and biogenesis (3), posttranslational modification, protein turnover, chaperones (3), and others.

A total of 12 proteins belonging to cellular metabolism and energy production were identified. Dramatic alterations in the protein expression during salt stress took place in T. halophilus. After salt shock, the expressions of proteins involved in amino acid transport and metabolism (spot 104), carbohydrate

Table 1 Identification of $T$. halophilus proteins affected by salt stress

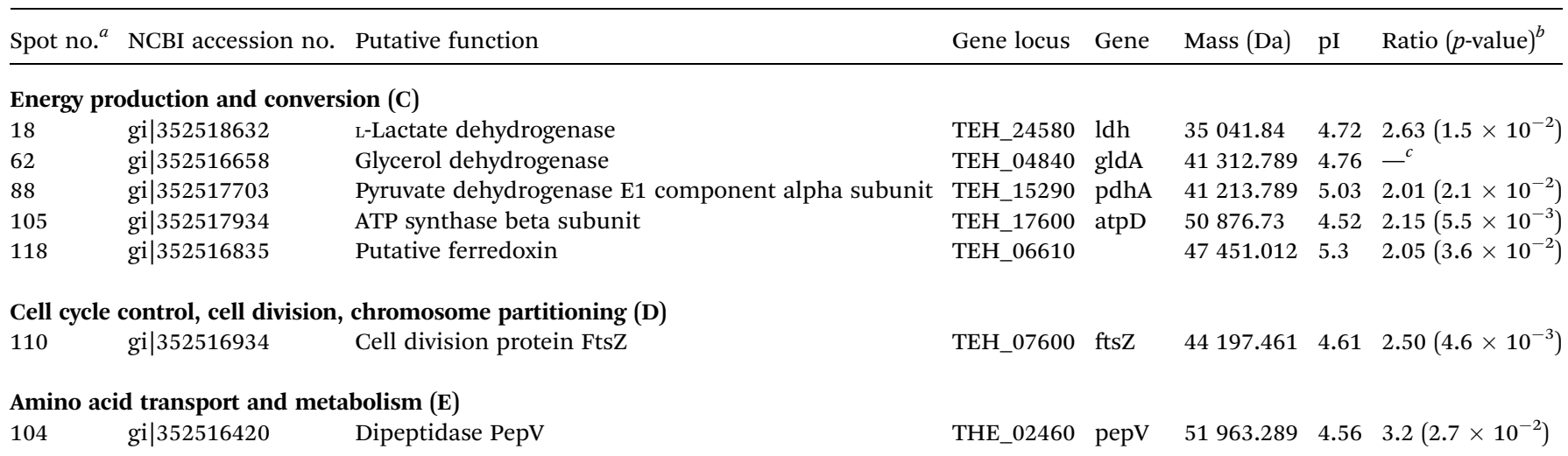

Carbohydrate transport and metabolism (G)

22 gi|352518123 Phosphoenolpyruvate-protein phosphotransferase

29 gi|352517161 Fructose-bisphosphate aldolase

97 gi|352517071 6-Phosphofructokinase

99 gi|352517949 Glyceraldehyde-3-phosphate dehydrogenase

111 gi|352517946 Enolase

112 gi|352517948 Phosphoglycerate kinase

Lipid transport and metabolism (I)

54 gi|352518404 Acyl-ACP-phosphate acyltransferase

Translation, ribosomal structure and biogenesis ( $\mathrm{J}$ )

$\begin{array}{lll}53 & \text { gi|352517495 } & \text { Tryptophanyl-tRNA synthetase } \\ 72 & \text { gi|352518461 } & \text { Elongation factor G } \\ 78 & \text { gi|352518460 } & \text { Elongation factor Tu }\end{array}$

Cell wall/membrane/envelope biogenesis (M)

14 gi|352516351 D-Alanine-D-alanine ligase

$47 \quad$ gi|352518617 $\quad N$-Acetylmuramic acid 6-phosphate etherase

81 gi|352518036 Glycine betaine ABC transporter ATP-binding protein
THE_19490 ptsI

THE_09870 fba

THE_08970 pfk

THE_17750 gap

TEH_17720 eno

TEH_17740 pgk
$63700.781 \quad 4.68 \quad 2.66\left(1.9 \times 10^{-2}\right)$ $31482.68 \quad 4.84 \quad 2.04\left(7.2 \times 10^{-3}\right)$ $34427.6295 .51 \quad 2.05\left(4.5 \times 10^{-3}\right)$ $\begin{array}{lll}35758.141 & 4.79 & 2.11\left(2.3 \times 10^{-2}\right)\end{array}$ $\begin{array}{llll}46 & 628.559 & 4.58 & 3.05 \\ \left(5.2 \times 10^{-3}\right)\end{array}$ $42176.762 \quad 4.912 .31\left(2.6 \times 10^{-3}\right)$

TEH_22300 plsX $35799.77 \quad 5.5 \quad 0.46\left(4.1 \times 10^{-3}\right)$

THE_13210 trpS $37431.121 \quad 5.54 \quad 0.39\left(1.9 \times 10^{-2}\right)$ TEH_22870 fusA $77058.273 \quad 4.83 \quad 0.34\left(2.2 \times 10^{-3}\right)$ THE_22860 tuf $\quad 43469.801 \quad 4.82 \quad 2.44\left(6.1 \times 10^{-3}\right)$

THE_01770 ddl $40276.32 \quad 4.87 \quad 0.47\left(1.2 \times 10^{-3}\right)$ THE_24430 murQ $32040.619 \quad 5.37 \quad 0.42\left(4.3 \times 10^{-3}\right)$ THE_18620 opuAA $47005.07 \quad 4.83 \quad 2.65\left(1.9 \times 10^{-3}\right)$

TEH_13000 dnaK $66743.43 \quad 4.38 \quad 3.68\left(3.6 \times 10^{-2}\right)$ TEH_02640 $\operatorname{trxB} \quad 34426.031 \quad 4.67 \quad$ - $^{c}$ TEH_11250 groEL $57740.68 \quad 4.47 \quad 3.05\left(1.5 \times 10^{-2}\right)$

TEH_16010 $\quad$ sodA $\quad 22700.051 \quad 5.06 \quad 2.04\left(3.6 \times 10^{-3}\right)$
THE_21640 $\quad 17457.9394 .65 \quad 0.45\left(4.5 \times 10^{-3}\right)$ $\begin{array}{lllll}\text { THE_17160 } & \text { cсpA } & 36383.988 & 5.17 & 0.37\end{array}\left(2.6 \times 10^{-2}\right)$
Posttranslational modification, protein turnover, chaperones $(0)$

1 gi|352517474 Chaperone protein DnaK

59 gi|352516438 Thioredoxin reductase

$73 \quad$ gi|352517299 $60 \mathrm{kDa}$ chaperonin

Inorganic ion transport and metabolism (P)

122 gi|352517775 Superoxide dismutase

Signal transduction mechanisms (T)

$\begin{array}{lll}44 & \text { gi } \mid 352518338 & \text { UspA family protein } \\ 86 & \text { gi|} 352517890 & \text { Catabolite control protein A }\end{array}$

${ }^{a}$ Spot numbers refer to the proteins labeled in Fig. $2 .{ }^{b}$ Fold change in intensity relative to salt stress. Values are means of at least three independent experiments. ${ }^{c}$ Proteins only detected during salt stress. 
metabolism and energy production (spots 18, 29, 88, 97, 105, $111,112,118$ ) were enhanced (Table 1). One protein (spot 54) involved in lipid transport, and two proteins (spots 53 and 72) involved in translation were down-regulated after salt stress. In addition, two proteins (spots 14 and 47) which is closely involved in peptidoglycan synthesis were detected, and a significantly down-regulated in protein expression was observed during salt stress. As expected, two classical stress shock proteins including chaperone protein Dnak (spot 1) and $60 \mathrm{kDa}$ chaperonin (spot 73) exhibited remarkably up-regulated. Whereas, UspA family protein (spot 44) and catabolite control protein A (spot 86) were markedly down-regulated (Table 1).

\subsection{Regulation of membrane fatty acid in T. halophilus during salt stress}

Effect of salt stress on the composition of membrane fatty acid was investigated (Fig. 4). As is shown, a total of 13 fatty acids were detected, and the major fatty acids were pentadecyclic acid (C15:0), palmitic acid (C16:0), stearic acid (C18:0), oleic acids (C18:1 n-9), and eicosanoic acid (C20:0), making up approximately $82.3 \%$ of the total fatty acids content of $T$. halophilus without salt stress. After salt shocked, the proportion of saturated fatty acids (SFA, C17:0, C18:0, and C19:0) decreased, and the proportion of unsaturated fatty acids (UFA, C16:1, C18:1, C18:2, and C18:3) increased. A detailed analysis of the ratio of UFA to SFA (U/S ratio) and mean chain length of the membrane fatty acids demonstrated that salt stress resulted in higher U/S ratio and mean chain length, compared to those without salt stress (Fig. 5).

\subsection{Changes of amino acid pools in $T$. halophilus during salt stress}

Effect of salt stress on the pools of amino acid was investigated. Significant difference was observed in the contents of 8 amino acids between cells with and without salt stress (Fig. 6). Salt stress induced a significant increase in the levels of proline and glycine, when cultured in GM17 containing $12 \% \mathrm{NaCl}$, and the levels of proline and glycine were 2.3-fold and 2.4-fold higher than those in cells grown without $\mathrm{NaCl}$, respectively. Whereas, the levels of threonine, valine, leucine, isoleucine, serine, and alanine decreased remarkably in the salt-stressed cells (Fig. 6).

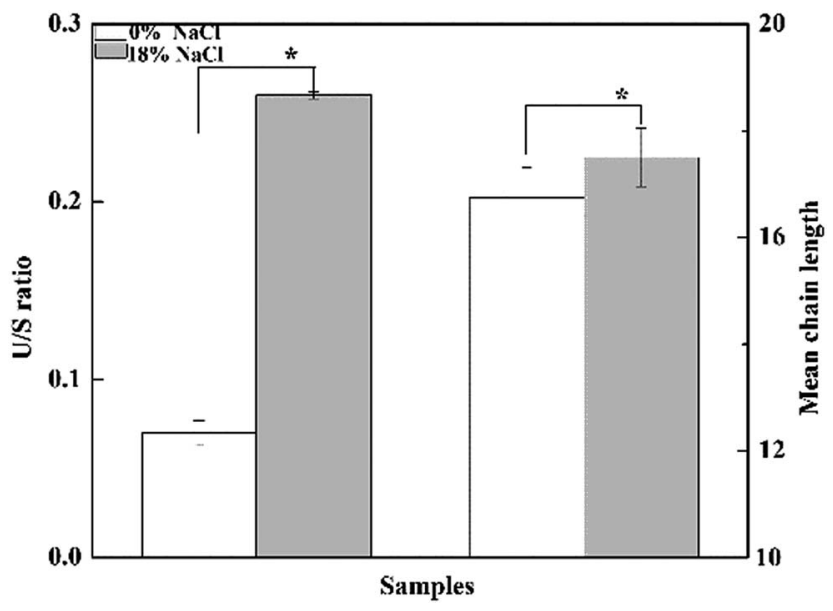

Fig. 5 Degree of unsaturation and mean chain length in T. halophilus under salt stress. The degree of unsaturation was calculated by unsaturated fatty acids/saturated fatty acids (U/S ratio). Asterisks indicate the level of statistical significance $(p<0.05, n=3)$ in comparison to the control $(0 \% \mathrm{NaCl})$.

\subsection{Accumulation of osmoprotectants in T. halophilus during salt stress}

To investigate the accumulation of osmoprotectants in T. halophilus during salt stress, alterations in intracellular concentrations of glycine betaine and trehalose were measured (Fig. 7). The levels of glycine betaine increased with the enhancement of $\mathrm{NaCl}$ concentration, and the maximum accumulation of glycine betaine (40.3 $\mathrm{mg} \mathrm{g}^{-1} \mathrm{DCW}$ ) was obtained at $12 \% \mathrm{NaCl}$ (Fig. 7A). Similar tendency was also observed in the content of trehalose (Fig. 7B). Salt stress led to the accumulation of trehalose, and a 1.9-fold higher amount of trehalose was detected at $9 \% \mathrm{NaCl}$ compared with that without $\mathrm{NaCl}$ addition. These results suggested that accumulation of glycine betaine or trehalose could play a potential protective role during salt stress.

\subsection{Expression levels of genes in T. halophilus during salt stress}

Transcriptional levels of $p y k, L d h, A t p D$, Dnak, GroEL, and opuAA in T. halophilus under salt stress were shown in Fig. 8. The
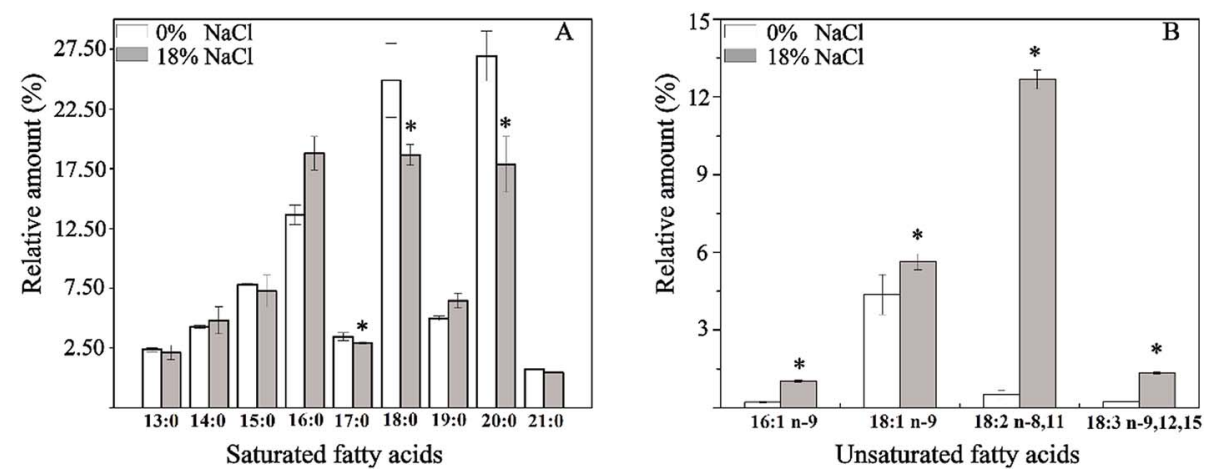

Fig. 4 Alteration in membrane fatty acids in T. halophilus under salt stress. Cells were exposed to the salt stress (18\% $\mathrm{NaCl}, 4 \mathrm{~h})$, and the proportion of membrane fatty acids ((A) saturated fatty acids, (B) unsaturated fatty acids) was determined. Asterisks indicate the level of statistical significance $(p<0.05, n=3)$ in comparison to the control $(0 \% \mathrm{NaCl})$. 



Fig. 6 Changes in intracellular pools of amino acids of T. halophilus under salt stress. (A) Proline, (B) glycine, (C) threonine, (D) valine, (E) leucine, (F) serine, $(G)$ isoleucine, $(H)$ alanine. Different letters indicate significant differences at $p<0.05(n=3)$.

expression levels of 6 genes were all up-regulated when cells were treated with $12 \% \mathrm{NaCl}$. Statistical analysis showed that the expression levels of salt-stressed cells significantly increased 5.4-, 4.7-, 1.3-, 14.9-, 8.3-, and 2.6-fold for pyk, Ldh, AtpD, Dnak, GroEL, and $о p u A A$, respectively, compared to those control cells. These results were agreement with the data of proteomic analysis.

\section{Discussion}

Salt stress is a commonly encountered environment for T. halophilus during the manufacture of fermented foods. The molecular basis for the salt tolerance is only partly understood, although numerous studies have been carried out to investigate the response and mechanism during salt stress. In this respect, 

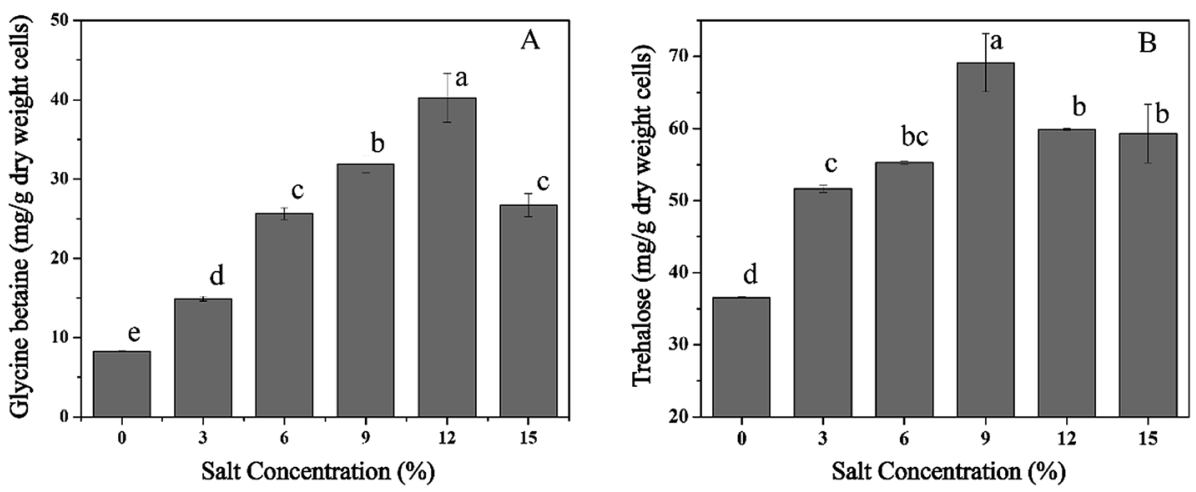

Fig. 7 Accumulation of glycine betaine and trehalose by T. halophilus in response to salt stress. (A) Glycine betaine, (B) trehalose. Different letters indicate significant differences at $p<0.05(n=3)$.

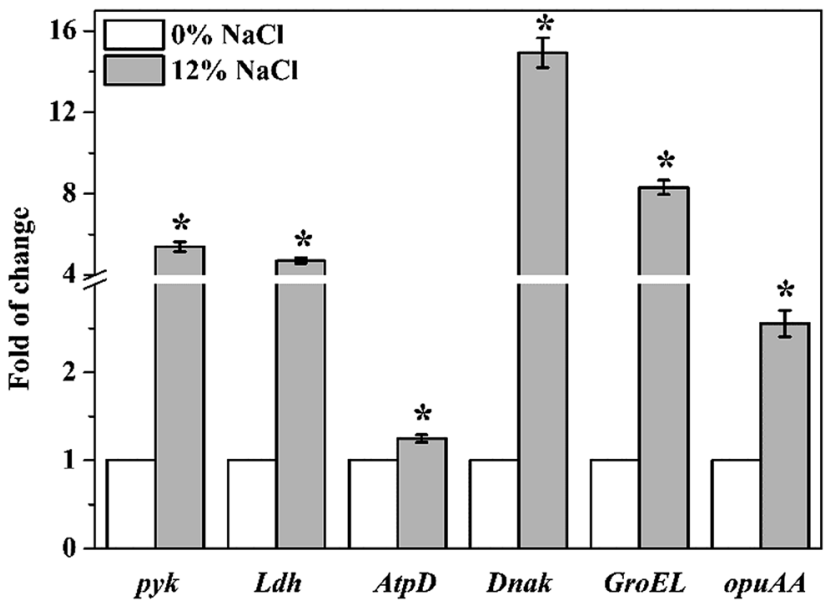

Fig. 8 Transcriptional levels of pyk, Ldh, AtpD, Dnak, GroEL, and opuAA in $T$. halophilus. Asterisk indicates significant differences with the corresponding control $(0 \% \mathrm{NaCl})$ at $p<0.05$ by the Student's $t$ test.

the proteome reference map is able to give a global picture of the relative abundance of proteins for microbes during physiological or environmental changes. In the present study, for the first time, a 2-DE proteome reference map of $T$. halophilus was constructred, and based on this, a combined physiological and comparative proteomic analysis was performed to further indepth elucidate the mechanisms to salt tolerance. A partial proteomic map of T. halophilus was constructed with a $\mathrm{pI}$ at $\mathrm{pH}$ $4-7$, where most proteins of lactic acid bacteria were located. A total of 120 proteins were identified (Table S2 $\dagger$ ), and the physico-chemical analysis was also performed (Fig. 3). Based on this proteomic data, comparative proteomic analysis was carried out to investigate the proteins implicated in salt tolerance.

\subsection{Changes in cell wall and cell membrane during salt stress}

Cell wall/membrane play important roles in cellular growth, metabolism, and resistance to environmental stresses, and cells fight against stresses by regulating the structure and compositions of wall/membrane. ${ }^{19}$ The adaptation of $T$. halophilus to high salt stress led to important variations in the distribution of membrane fatty acids. Cells treated with $18 \%$ $\mathrm{NaCl}$ exhibited a significant increase in the levels of unsaturated fatty acids and a decrease in the proportion of saturated fatty acids (Fig. 4 and 5). These results are similar to those reported in other bacteria, ${ }^{31,32}$ suggesting that modulation of the fatty acids composition is necessary for survival during salt stress. In addition, increase of the ratio of UFA/SFA can protect the cell from other environmental stresses in LAB has also been observed. ${ }^{19,33}$ Furthermore, higher mean chain length of the membrane fatty acid was observed in T. halophilus shocked by salt stress (Fig. 5), and similar results were also reported in $L$. casei, Streptococcus gordonii, and Streptococcus salivarius treated by acid stress. ${ }^{19,34}$

Analysis of the proteomic data showed that the expressions of D-alanine-D-alanine ligase (spot 14) and $N$-acetylmuramic acid 6-phosphate etherase (spot 47), which were involved in the biosynthesis of peptidoglycan were significantly downregulated when challenged by high salt stress (Fig. 2), and this result was in agreement with that reported by Liu et al. ${ }^{14}$ Previous researches suggested that peptidoglycan played a positive role in stress resistance under low salt stress, while the synthesis of peptidoglycan was reduced to promote ion transport across extra- and intracellular membranes under high salt condition. ${ }^{14,35}$

\subsection{Accumulation of osmoprotectants upon salt stress}

Amino acid metabolism serves a number of physiological roles including intracellular $\mathrm{pH}$ control, resistance to stresses, and generation of metabolic energy or redox power. ${ }^{36}$ Previous researches have indicated that many amino acids or their derivatives may protect cells against salt stress. Zhang et al. ${ }^{11}$ investigated the effect of glutathione addition on survival of $L$. lactis during salt stress $(5 \mathrm{M} \mathrm{NaCl})$, and the results showed that the presence of $3.2 \mathrm{mM} \mathrm{GSH}$ in the medium resulted in a more than 2-log increase in cell survival after $60 \mathrm{~min}$ incubation, and greater than 3-log improvement after $2 \mathrm{~h}$. In this study, changes in contents of intracellular amino acids were investigated, and 8 amino acids exhibited significantly difference between cells 
treated with and without salt stress (Fig. 6), suggesting that these amino acids may contribute to the osmoregulation in $T$. halophilus. Glycine is the precursor of glycine betaine, ${ }^{7}$ and higher content of glycine may result in higher production of glycine betaine, and this result is in agreement with that obtained by intracellular glycine betaine determination in Fig. 7.

In addition, it was worth noting that the opuAA gene and glycine betaine $\mathrm{ABC}$ transporter protein (OpuAA, spot 81) were also significantly up-regulated during salt treatment (Fig. 8 and Table 1). Proline is accumulated by a large number of LAB under osmotic stress as an osmoprotectant including Pediococcus pentosaceus, ${ }^{37}$ and L. lactis $^{38}$ In particular, it can act as a signaling molecule to influence defense pathways and relieve the osmotic stress caused by salinity. In addition, previous data suggested that a negative correlation between salt stress and serine, valine, threonine, and isoleucine was achieved in Synechocystis sp. PCC 6803 via metabolomic analysis, ${ }^{39}$ and the result was in agreement with our data.

Compatible solutes are low-molecular weight osmoregulatory compounds, including sugars, amino acids, quaternary amines, ectoines or their derivatives. ${ }^{40}$ Induction of osmolytes in cells can increase protein folding and thereby improve salt tolerance. Accumulation of glycine betaine allows the cells to withstand salt stress and protect cells against dehydration. In this study, the contents of glycine betaine in T. halophilus cells increased with increasing $\mathrm{NaCl}$ levels (Fig. 7). These results are in agreement with previous findings confirming salinity induced glycine betaine accumulation., ${ }^{7,38,41,42}$ In addition, trehalose is a frequent compatible solute in bacteria, fungi, and plants, and the protective role of trehalose in a variety of stresses has been abundantly demonstrated. ${ }^{\mathbf{4 3 , 4 4}}$ In the present study, similar result was also obtained during salt stress, suggesting that trehalose was an important osmoprotectant for $T$. halophilus in salt adaptation.

\subsection{Regulation of cellular metabolism under salt stress}

Cellular metabolism plays an important role during the $\mathrm{NaCl}-$ induced regulation in cells. For example, carbohydrate metabolism generates ATP to maintain the intracellular microenvironment homeostasis, which includes ion transport, redox balance, and osmoregulation. ${ }^{14}$ In this study, salt stress led to the overproduction of many proteins involved in cellular metabolism and energy production, especially proteins in glycolysis. The increase in protein expression may be the integrant to overcome the inhibition in cells challenging at high salt condition. Then, the increased flux in cellular metabolism may lead to an increase in ATP production to support ATP-demanding processes such as $\mathrm{H}^{+}$extrusion and osmoregulation. To further verify these results, we measured the contents of lactic acid, citric acid, and succinic acid (Fig. 9). As is shown, the contents of lactic acid and citric acid increased with the increase of salinity, whereas, the amount of succinic acid decreased with increased $\mathrm{NaCl}$ content. As mentioned above, salt stress resulted in the accumulation of proline, and the synthesis of proline requires a continuous supply of the precursor $\alpha$-oxoglutarate and glutamate. Thus,
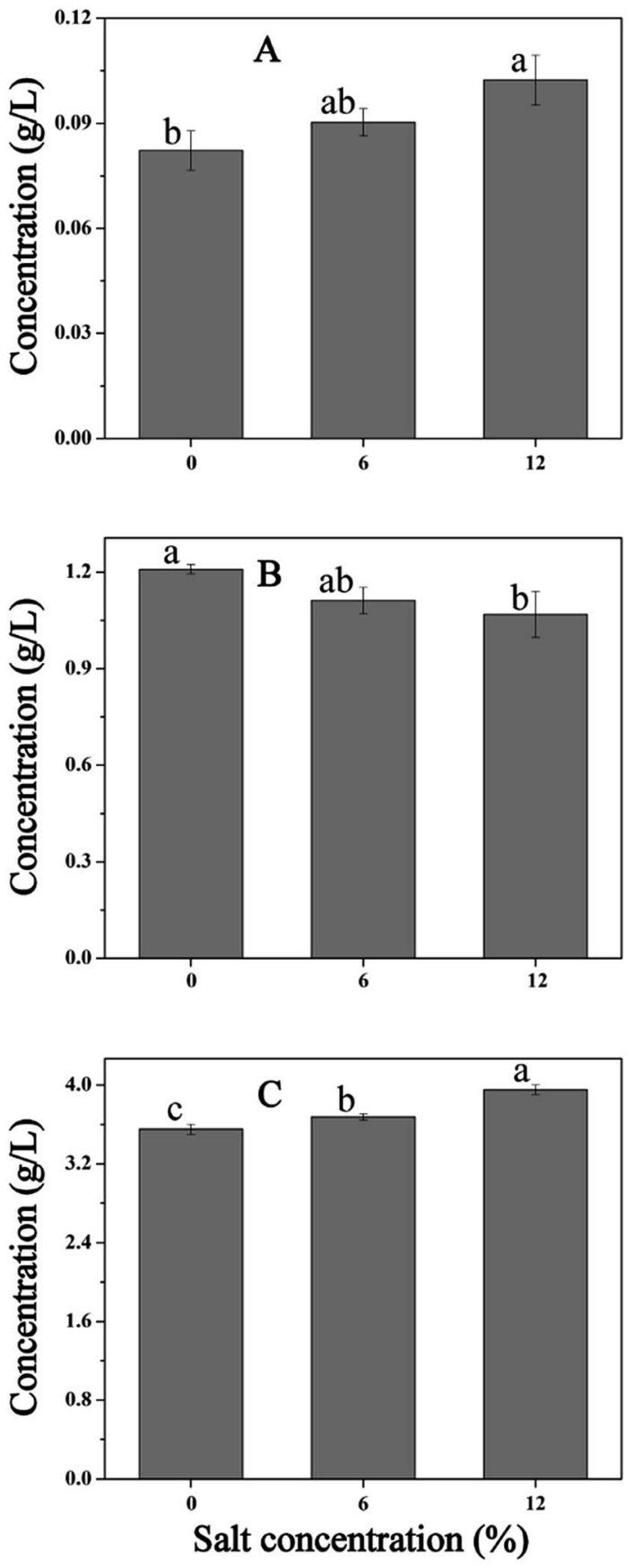

Fig. 9 Changes in contents of organic acids of T. halophilus under salt stress. (A) Citrate acid, (B) succinate acid, (C) lactate acid. Different letters indicate significant differences at $p<0.05(n=3)$.

the increased content of citric acid and decreased content of succinic acid may contribute to the production of proline. Hahne et al. ${ }^{\mathbf{4 4}}$ performed by proteomic and transcriptomic analysis, which suggested that the enzymes of tricarboxylic acid (TCA) cycle leading to the synthesis of $\alpha$-oxoglutarate were induced and the reactions from $\alpha$-oxoglutarate to oxaloacetate were reduced. Moreover, the increased expression of gene AtpD and AtpD protein (spot 105) under salt stress also confirmed this result. 
4.4. General stress proteins and response factor induced by salt stress

Four general stress response proteins (DnaK, GroEL, SodA, and TrxB) were significantly expressed $(p<0.05)$ during salt stress. Up-regulation of genes Dnak and GroEL performed by q-PCR also confirmed this result. The proteins Dnak and GroEL are known to protect or repair proteins under stress conditions. DnaK is believed to serve as a "cellular thermometer" that transduces signals to other cellular factors in response to environmental stresses, and it required for stress tolerance. ${ }^{45}$ The production of DnaK also increased after salt stress in T. halophilus ${ }^{\mathbf{1 4}}$ and L. lactis. ${ }^{\mathbf{4 6}}$ Moreover, Sugimoto et $a l .{ }^{47}$ heterologously expressed $d n a K$ from $E$. coli in L. lactis NZ9000, and improvement of multiple-stress tolerance and lactic acid production was observed. Likely, the overproduction of GroEL under salt stress was also reported in previous researches, ${ }^{\mathbf{1 4}, 46}$ and the GroESL-overproducing $L$. lactis and L. paracasei exhibited higher tolerance to heat, salt, and butanol stresses. ${ }^{48}$

Both superoxide dismutase (SodA) and thioredoxin reductase (TrxB) are responsible for dealing with imbalances in redox state and reactive oxygen species (ROS). SodA, involved in the defense against ROS was induced during salt stress. Generally, salt stress leads to changes in the osmotic pressure and ionic inequity, which causes production of ROS thereby also triggers an oxidative stress. Interestingly, salt stress induced production of SodA was also detected in Enterobacter sakazakii and Synechocystis sp. strain PCC 6803. ${ }^{49,50}$ Thus, the overproduction of SodA may protect cells against ROS that might be produced as by-products or accumulate due to respiratory chain impairment or metabolic alterations during stress situations. ${ }^{51}$ As expected, a significantly higher expression of thioredoxin reductase (TrxB) $(p<0.01)$ was observed during salt stress in this study, and similar results were also reported previously. ${ }^{\mathbf{1 4 , 4 9}}$

\section{Conclusion}

In conclusion, a reference proteome map of T. halophilus was established by using a proteomic approach. For the first time we described the proteome of T. halophilus and analyzed the COG, CAI, and GRAVY values of each identified protein compared with the whole theoretical genes coding proteins of $T$. halophilus. By comparing the proteomic profile and physiological characterization of $T$. halophilus during high salt condition, we further demonstrated the stress response of cells to defend against salt stress. In response to salt stress, T. halophilus evokes a global regulation and a number of changes take place. These results may help us understand salt tolerance mechanism and help formulate new strategies to enhance the industrial application of this species.

\section{Acknowledgements}

This study was funded by the National Natural Science Foundation of China (31671849, 31301546).

\section{References}

1 C. Wu, C. Liu, G. He, J. Huang and R. Zhou, Food Sci. Technol. Res., 2013, 19, 855-864.

2 W. Roling and H. Van Verseveld, Appl. Environ. Microbiol., 1996, 62, 1203-1207.

3 N. Udomsil, S. Rodtong, S. Tanasupawat and J. Yongsawatdigul, Int. J. Food Microbiol., 2010, 141, 186-194.

$4 \mathrm{~J}$. Thongsanit, S. Tanasupawat, S. Keeratipibul and S. Jatikavanich, Japanese Journal of Lactic Acid Bacteria, 2002, 13, 46-52.

5 R. Cui, J. Zheng, C. Wu and R. Zhou, Eur. Food Res. Technol., 2014, 239, 321-331.

6 J. M. Wood, J. Gen. Physiol., 2015, 145, 381-388.

7 H. Robert, C. Le Marrec, C. Blanco and M. Jebbar, Appl. Environ. Microbiol., 2000, 66, 509-517.

8 M. E. Guerzoni, R. Lanciotti and P. S. Cocconcelli, Microbiology, 2001, 147, 2255-2264.

9 C. Kohler, R. F. Lourenço, J. Bernhardt, D. Albrecht, J. Schüler, M. Hecker and S. L. Gomes, BMC Microbiol., 2015, 15, 1-15.

10 C. Rubianolabrador, C. Bland, G. Miotello, P. Guérin, O. Pible, S. Baena and J. Armengaud, J. Proteomics, 2014, 97, 36-47.

11 Y. Zhang, Y. Zhang, Y. Zhu, S. Mao and Y. Li, Appl. Environ. Microbiol., 2010, 76, 3177-3186.

12 C. Wu, J. Zhang, G. Du and J. Chen, Bioresour. Technol., 2013, 143, 238-241.

13 X. Dong, B. Tian, S. Dai, T. Li, L. Guo, Z. Tan, Z. Jiao, Q. Jin, Y. Wang and Y. Hua, PLoS One, 2014, e10.

14 L. Liu, L. Si, X. Meng and L. Luo, J. Ind. Microbiol. Biotechnol., 2015, 42, 601-616.

15 G. He, C. Wu, J. Huang and R. Zhou, Process Biochem., 2016, 51, 213-219.

16 T. Michelson, K. Kask, E. Jõgi, E. Talpsep, I. Suitso and A. Nurk, Enzyme Microb. Technol., 2006, 39, 861-867.

17 M. Jebbar, G. Gouesbet, S. Himdi-Kabbab, C. Blanco and T. Bernard, Arch. Microbiol., 1995, 163, 380-386.

18 S. S. T. Hua, B. J. Hernlem, W. Yokoyama and S. B. L. Sarreal, World J. Microbiol. Biotechnol., 2015, 31, 729-734.

19 C. Wu, J. Zhang, M. Wang, G. Du and J. Chen, J. Ind. Microbiol. Biotechnol., 2012, 39, 1031-1039.

20 J. Zheng, R. Liang, L. Zhang, C. Wu, R. Zhou and X. Liao, Food Res. Int., 2013, 54, 660-666.

21 C. Wu, G. He and J. Zhang, J. Ind. Microbiol. Biotechnol., 2014, 41, 1533-1540.

22 S. Mao, Y. Luo, T. Zhang, J. Li, G. Bao, Y. Zhu, Z. Chen, Y. Zhang, Y. Li and Y. Ma, J. Proteome Res., 2010, 9, 30463061.

23 A. Guillot, C. Gitton, P. Anglade and M. Y. Mistou, Proteomics, 2003, 3, 337-354.

24 K. Hiller, M. Schobert, C. Hundertmark, D. Jahn and R. Münch, Nucleic Acids Res., 2003, 31, 3862-3865.

25 R. Wu, X. Song, Q. Liu, D. Ma, F. Xu, Q. Wang, X. Tang and J. Wu, Ann. Microbiol., 2016, 1-8. 
26 K. J. Livak and T. D. Schmittgen, Methods, 2001, 25, 402408.

27 K. Fukami, Y. Funatsu, K. Kawasaki and S. Watabe, J. Food Sci., 2004, 69, 45-49.

28 Y. Tanaka, J. Watanabe and Y. Mogi, Food Microbiol., 2012, 31, 100-106.

29 R. Wu, W. Wang, D. Yu, W. Zhang, Y. Li, Z. Sun, J. Wu, H. Meng and H. Zhang, Mol. Cell. Proteomics, 2009, 8, 2321-2338.

30 J. Yuan, L. Zhu, X. Liu, T. Li, Y. Zhang, T. Ying, B. Wang, J. Wang, H. Dong and E. Feng, Mol. Cell. Proteomics, 2006, 5, 1105-1118.

31 S. I. Allakhverdiev, Y. Nishiyama, I. Suzuki, Y. Tasaka and N. Murata, Proc. Natl. Acad. Sci. U. S. A., 1999, 96, 5862-5867.

32 C. Lopez, H. Heras, H. Garda, S. Ruzal, C. Sanchez-Rivas and E. Rivas, Int. J. Food Microbiol., 2000, 55, 137-142.

33 Y. Wang, G. Corrieu and C. Béal, J. Dairy Sci., 2005, 88, 21-29.

34 E. M. Fozo, J. K. Kajfasz and R. G. Quivey, FEMS Microbiol. Lett., 2004, 238, 291-295.

35 K. J. Cheung, V. Badarinarayana, D. W. Selinger, D. Janse and G. M. Church, Genome Res., 2003, 13, 206-215.

36 M. Fernández and M. Zúñiga, Crit. Rev. Microbiol., 2006, 32, 155-183.

37 A. Baliarda, H. Robert, M. Jebbar, C. Blanco, A. Deschamps and C. L. Marrec, Int. J. Food Microbiol., 2003, 84, 13-20.

38 D. Obis, A. Guillot, J. C. Gripon, P. Renault, A. Bolotin and M. Y. Mistou, J. Bacteriol., 1999, 181, 6238-6246.
39 J. Wang, X. Zhang, M. Shi, L. Gao, X. Niu, R. Te, L. Chen and W. Zhang, Funct. Integr. Genomics, 2014, 14, 431-440.

40 B. Kempf and E. Bremer, Arch. Microbiol., 1998, 170, 319330.

41 M. Jebbar, C. Blanco and C. Le Marrec, Curr. Microbiol., 2003, 47, 347-351.

42 A. Metris, S. M. George, F. Mulholland, A. T. Carter and J. Baranyi, Appl. Environ. Microbiol., 2014, 80, 4745-4756.

43 S. B. Leslie, E. Israeli, B. Lighthart, J. H. Crowe and L. M. Crowe, Appl. Environ. Microbiol., 1995, 61, 35923597.

44 H. Hahne, U. Mäder, A. Otto, F. Bonn, L. Steil, E. Bremer, M. Hecker and D. Becher, J. Bacteriol., 2010, 192, 870-882.

45 P. Carranza, A. Grunau, T. Schneider, I. Hartmann, A. Lehner, R. Stephan, P. Gehrig, J. Grossmann, K. Groebel, L. E. Hoelzle, L. Eberl and K. Riedel, Proteomics, 2010, 10, 3248-3261.

46 M. Kilstrup, Appl. Environ. Microbiol., 1997, 63, 1826-1837.

47 S. Sugimoto, C. Higashi, S. Matsumoto and K. Sonomoto, Appl. Environ. Microbiol., 2010, 76, 4277-4285.

48 C. Desmond, G. F. Fitzgerald, C. Stanton and R. P. Ross, Appl. Environ. Microbiol., 2004, 70, 5929-5936.

49 S. Fulda, S. Mikkat, F. Huang, J. Huckauf, K. Marin, B. Norling and M. Hagemann, Proteomics, 2006, 6, 27332745 .

50 K. Riedel and A. Lehner, Proteomics, 2007, 7, 1217-1231.

51 G. Cacace, M. F. Mazzeo, A. Sorrentino, V. Spada, A. Malorni and R. A. Siciliano, J. Proteomics, 2010, 73, 2021-2030. 